\title{
ANIMA Noise Platform and ANIMA Methodology: One-Stop Shop for Aviation Noise Management
}

\author{
Alexandra Covrig and G. Heyes $\mathbb{D}$
}

\begin{abstract}
When you think about aviation noise, you might imagine an airplane taking off. When you think about decreasing aviation noise, the first thing that usually comes up in one's mind are the new silent plane engines. This makes perfect sense, but it does not fully grasp the issue of aviation noise. The ANIMA project is based on a holistic approach to aviation noise, as it focuses on non-acoustical factors as well. Annoyance, as perceived by local communities surrounding airports, also depends on non-acoustical factors, which can be situational (time of the day, day of the week, activity performed while exposed to noise) and personal (sensitivity to noise, attitudes, noise insulation).
\end{abstract}

Keywords Airport communities • Communication • Community engagement • Design thinking

\section{How is ANIMA Different and What is its Added Value}

While seeking to better understand annoyance, ANIMA observed that aviation noise is not only an engineering issue which requires reducing noise at source. Reducing annoyance only by using quieter aircraft is indeed helpful, but not enough to make an airport a good neighbour to the local residents. Therefore, ANIMA takes a different and innovative stance to aviation noise. The project carried out research from an

Illustrating How ANIMA is Endeavouring to Propose a One-Stop Shop Where Various Aviation Noise-Related Stakeholders Would Find Possible Solutions for the Challenges They Are Confronted with.

\footnotetext{
A. Covrig $(\varangle)$

Airport Regions Council, Rue Montoyer 21, 1000 Brussels, Belgium

e-mail: alexandra.covrig@airportregions.org

G. Heyes

Ecology and Environment Research Centre, Department of Natural Sciences, Manchester Metropolitan University, Chester Street, Manchester M1 5GD, UK

e-mail: g.heyes@mmu.ac.uk
} 
interdisciplinary approach, by bringing together aircraft engineers, urban geographers, psychologists, sociologists, noise experts and regulatory experts. This diverse and comprehensive partnership resulted in novel approaches to aviation noise impact management and mitigation. ANIMA is not the traditional aviation noise research project, since it aimed at complementing the existing intensity-averaged noise maps by dynamic profile-dependent annoyance maps.

In addition to its novel approach to aviation noise impact management, the holistic character of ANIMA stems also from the fact that it builds on other previous projects, such as X-NOISE, SEFA (Sound Engineering For Aircraft), COSMA (Community Oriented Solutions to Minimise aircraft noise Annoyance) and TEAM PLAY. XNOISE was a Coordination and Support Action project which focused on aircraft noise and on lowering the noise exposure of communities. The project coordinated research activities and created an aero-acoustical knowledge base. ANIMA not only maintained the legacy of X-NOISE, but it extended its role through the setup of a specific committee gathering other relevant EU project coordinators. It also developed and consolidated a scenario-based, impact-driven strategic roadmap for aviation noise research. Throughout its four years of activity, ANIMA has been successfully leading the global coordination of European research efforts on aviation noise and it encouraged the wider network of experts and stakeholders, at both European and national levels, to maintain and enrich the roadmap developed within the project. As for SEFA, it was the pioneering EU project on aircraft noise impact. It included laboratory hearing tests and started developing the Virtual Resident tool. ANIMA followed up on SEFA by further developing the tool into a new inclusive version: Virtual Community Tool. This version comprises new scenarios with more accurate and thorough behavioural reactions to aircraft noise. The tool allows users to test traffic around airports as well as possible evolutions with new aircraft or flight scenarios. After SEFA, COSMA further built on this project, as it focused on laboratory tests and field investigations on noise in order to develop engineering criteria for aircraft design and operations that help reduce annoyance. ANIMA expanded the scope of COSMA, by exploring management and community engagement, rather than looking at noise itself. Regarding TEAM PLAY, the project created a modelling framework to support the European perspective in the international policy arena. ANIMA added to this framework an augmented modelling capability related to annoyance and a noise management toolset designed to enable use by the wider audience.

Looking at the development process of ANIMA, it can be noticed that there are two underlying elements at the core of the project: non-acoustical factors and communication. When addressing annoyance, non-acoustical factors and communication are at the centre of ANIMA's unique approach. 


\section{Community Engagement in ANIMA Project}

Noise management in airport areas can only be successful if all parties, including the ones contributing to noise and those who can hear the noise, are engaged in dialogue at the same table and benefit from a common understanding of what is at stake.

Interventions meant to reduce annoyance should be designed based on structured exchanges with communities. Lack of transparent communication and fair exchanges usually lead to failed interventions, since the needs and expectations of the diverse stakeholders tend to differ. ANIMA understood that different stakeholders have different needs and there is no universal solution, but open dialogue can pave the way towards consensus. Structured exchanges between airports and communities must be fair, meaning that communities must benefit from distribution of userfriendly information which avoids technical jargon and that the standpoints of all stakeholders are taken into account. To reach consensus, shared and restored trust are prerequisites.

In this sense, ANIMA engaged with several local communities, such as residents from Gava (Spain), Iasi (Romania), Brussels (Belgium) and Kranj (Slovenia). Thanks to these numerous meetings and interactions, it became clear that there are still many knowledge gaps among relevant stakeholders. From these encounters, ANIMA learnt that, when coping with aviation noise, some residents feel "helpless", "left-out" or "unaware". In these circumstances, open and fair dialogue, which ensures equally-beneficial outcomes for everyone, can only occur when everyone is equipped with the same knowledge and understanding. To discuss aviation noise, pre-existing knowledge about a broad spectrum of different fields, such as aviation noise management, airport management, avionics, noise and exposure, health impacts and human behaviour, legislation, policymaking, might be necessary. No one can be an expert in everything, so there is a need in finding and understanding the missing parts of the noise puzzle. To facilitate engagement, communication must be inclusive, transparent and most-importantly, it must be a two-way process, where all parties can add to the dialogue. If a fair, inclusive and transparent decision-making process is set up with all stakeholders, including neighbouring communities, then authorities and airports must be ready to accept and endorse the consensus reached through the process. The goal is to develop a common noise policy for impact mitigation.

The main takeaway that can be drawn from the ANIMA events is that when it comes down to noise management, prevention and proactivity are key. If legislation is not yet available at the degree of needed protection, initiatives to increase quality of life must still be taken at national, regional and municipal level. Oftentimes, the level of awareness is not the same among stakeholders, hence the importance of working collaboratively towards common noise policy which benefits all parties. Better awareness and knowledge on different noise sources and indicators would support the understanding of the impact that noise has on human health and wellbeing in different ways. 


\section{ANIMA Noise Platform}

As previously stated, noise is not only a technical issue, but also a social, regulatory and political issue. To mitigate impact, stakeholders, ranging from policymakers to residents and from airports to manufacturers, have to work together towards solutions.

To encourage collaborative decision-making on the development of common noise policies and to ensure engagement with local communities, ANIMA project created a Noise Platform. This platform provides the medium and tools for stakeholders to address the challenge of aviation noise exposure. The Noise Platform is the result of the successful collaboration between aircraft and airport engineers, noise specialists, urban geographers, psychologists, sociologists and experts on aviation regulation, who sought to better understand the annoyance and to develop best practice solutions to alleviate this burden. The ANIMA partners carried out research and engaged in dialogue with policymakers, airports, and noise-affected communities to assess how aviation noise mitigation interventions are implemented and how affected these measures are in reducing annoyance and improving quality of life. This platform captures the results of this research, offering an overview of aviation noise regulation and how to implement it, as well as current gaps and new solutions to bridge those gaps. It also provides tools, such as a mobile application, which is meant to help airports and authorities to capture how local communities perceive annoyance. A Noise Management Toolset was also developed and is available on this platform. This toolset aims to help airports and authorities to compute noise maps and awakening indexes in order to test the impact of different scenarios with various fleet configurations and flights. In addition, the platform gives access to an enriched Aviation Noise Research Roadmap, which supports policymakers in defining future policy and research goals. Other ANIMA results are available on the platform, such as scientific publications summarising key findings on how to address aviation noise impact as well as more tools fostering community engagement and building transparent working relations across all stakeholders. The ANIMA Noise Platform is open to everybody, featuring free and user-friendly content.

The ANIMA Noise Platform was designed and built by applying and following the principles used in the ANIMA methodology of noise interventions and management, which will be covered in subsections below.

\section{'Designing' Effective Noise Management Measures}

At its core, noise management is a process of problem solving. It sees airports seeking to provide their service (facilitating air transport) in a way that causes as little noise impact as possible on residents, via a range of noise management measures (i.e. those described under the ICAO Balanced Approach elements), including 'people issues', or communication and engagement. Airports do this due to legislation that exists to protect residents from the health impacts of noise, or as part of their social-license to 
operate, by demonstrating to residents that they are doing all that they can to minimise noise, and thus reduce the likelihood for complaints that otherwise can constrain airport activity. In this sense, noise management actions and interventions can also be seen as services. They are things that the airport provides for the benefit of external beneficiaries, and hence the perceived success of those interventions depends very much on understanding and responding to the needs, demands, and wishes of those stakeholders. It is for this reason why, as described in Chap. 11, considering those perspectives, through communication and engagement is so crucial to the design of effective noise management strategies.

Most business activity is not actively designed (Downe, 2020). This is the case whether it be at the process or system level, the business model level, or the strategic level. Rather, the things that businesses do tend to organically evolve over time as individual problems arise and are solved in turn. The result can be a mesh of organisational processes and strategies that have never actually been holistically designed in a systematic or targeted way, instead, they essentially exist to solve problems (either for the business or for stakeholders), often with increasing levels of complexity or bureaucracy.

If most services are not designed, it poses the natural question of how design principles and processes can help to inform their development. Indeed, there is now an entire industry dedicated to concepts such as Design Thinking and Service Design, supported by a range of academic research and publications, including a focus on the management of environmental management issues such as noise. Design and design thinking concepts have also been applied in a range of aviation contexts, including challenges related to airport and air cabin design, safety, and security and by organisations such as airlines, the military, and NASA (Hall et al. 2013; Goodheart 2016; This Is DesignThinking.net, 2016; McGowan et al. 2017; Turner Donald 2017; Design For Europe 2020). In terms of noise design-led approaches are also advocated in national guidance such as the United Kingdom's Civil Aviation Authority CAP1616 document and the United States Federal Aviation Authority Program 150 document, which both use design process and principles to inform the airspace design.

Below, the concept of design is introduced and its potential role in noise management is described. We then present the ANIMA Method for designing and delivering noise management interventions, devised through a series of case studies with airports from across European Member States.

\section{Design-Led Approaches to Noise Management}

The wide range of characteristics that define each airport and the challenges that they face means that there is no ubiquitous solution to noise that can be applied to all airports. Instead, airports need to design their own tailored solutions to the distinctive challenges that they face. They need robust yet flexible approaches that 
can take them from the challenges that comprise a range of unknowns, to the development and implementation of solutions that solve core issues based on a deep understanding of the challenges faced. As ANIMA research has shown, and as described in Chap. 11, this process should embed concepts of stakeholder engagement and two-way dialogues that develop empathy for residents, and to develop outcomes that are perceived as fair by, where possible, incorporating elements of public participation into decision-making processes. Developing noise management interventions should also seek out measures that are able to address core problems that trigger (for instance) complaints rather than seeing complaints as the challenge to be solved. In other words, they should address the cause of noise issues rather than the symptom.

Importantly, noise management measures, to be truly effective, have to comply with three requirements. They must be:

- Viable in terms of complex factors such as safety, security, environmental interdependencies and legislative compliance;

- Feasible in terms of airport infrastructure and financial capabilities; and

- Desirable to industry and community stakeholders.

This sort of thinking can be incredibly helpful in empowering organisations to understand not just what is possible and best suited to their own needs, but also to those of their stakeholders. This is of critical importance for noise management where the perspectives of those for whom noise management measures are designed to benefit can have a significant influence on the perceived success of those measures. This is particularly the case considering the important role of non-acoustic factors that are referred to throughout this book. ANIMA research has shown that airports have historically performed well in terms of feasibility and viability as these are generally technical driven considerations that the industry has been managing for many decades. These criteria are complex, but they can be informed by quantitative monitoring and modelling data that can act as an evidence base to support and communicate the decision-making process to stakeholders, and to evidence the success of given measures. The desirability of noise management decisions, i.e. in the eyes of stakeholders, is however a much more challenging concept as it inherently requires the collection of qualitative data that requires a particular set of skills, can be time-consuming and involves a range of complex and conflicting viewpoints. Design-led approaches can help in this regard. 'Design thinking' is a human-centred (van der Bijl-Brouwer and Dorst 2017) and iterative step-approach to solving problems that acknowledges that there are many levels of understanding required to solve the complex challenges faced by organisations and that these perspectives must be considered for outcomes to be regarded as successful. Design thinking proposes an iterative process through which it is possible to move from a hypothetical starting position with many unknowns, towards solutions built on the needs of those who end-users by addressing the root cause of the problem at hand. It does this not by providing a rigid set of rules and actions. Instead, design thinking creates a series of spaces in which different types of activities take place (Brown 2008; Liedtka 2015), 
for example, empathy building, learning, brainstorming, trialling and implementation. As a flexible process, there is no agreed definition of what design thinking looks like, but generally, it is described as comprising four key phases:

- Discovery: A research phase in which an initial challenge or problem is explored by researchers in order to obtain a range of data (quantitative and qualitative in nature) that can be used to get a deeper understanding of the problem at hand. For noise management, this may include the monitoring and modelling of noise data, but also speaking to industry stakeholders (i.e. national airspace providers and airlines) and community stakeholders (i.e. local authority and community members) for their important and essential insight.

- Defining: An interpretative phase where the collected data is analysed with the aim of providing key insights into problems faced.

- Design: With a deeper level of insight, creative brainstorming design exercises take place to identify as many potential solutions as possible. Consultation events and the use of dialogue forums and other community groups is an increasing trend where design options and their selection are increasingly informing decision making in aviation.

- Delivery: The most promising solutions can be implemented through iterative processes of testing and trials, to understand the validity and likely impacts of the designed solutions, and their potential scaling up to full deployment. This is similar to approaches already undertaken in aviation where trialling of, for example, operational procedures typically takes place before full implementation.

As shown above, the aviation industry often conducts each of these phases as important activities, however, there does not exist any standardised approach that can help airports move through these processes in a systematic way that can be evidenced to stakeholders. Design thinking is one such way and has informed the development of the ANIMA Methodology described below.

\section{The ANIMA Methodology}

Design Thinking is similar to an approach described in ANIMA research to guide airports in developing noise management measures. The work posited that effective noise management follows a similar multi-step iterative process, which poses a range of questions. These steps and a range of example questions are provided below.

\section{Identification of the Need for an Intervention}

At this stage, an airport becomes aware of the need to implement a noise management measure. This means that airports should seek to learn as much about the situation as possible to help inform decision making processes and the ultimate design of the 
measure. The idea for an intervention may arise from a number of sources. Perhaps a new technology has become available, such as performance-based navigation, that the airport wishes to implement. It could be that communities have identified an opportunity for a change, or have been campaigning for a change in airspace design. An airport could be seeking to build new infrastructure or be responding to legislation. The key thing to consider here is to not take the initial identification at face value, but rather seek to understand as much as possible about the change. To understand, for example, if communities are complaining, what the reasons for those complaints are, and seeking to understand if the proposed changes will adequately make a positive difference. Stakeholders who have influence over the potential changes should be spoken with and engaged in a two-way dialogue-this, importantly, includes residents. Helpful questions and thinking at this stage may include:

- Do you have multi-stakeholder and independently led stakeholder engagement pathways (including community representatives) through which the requirement for an operational change could be communicated and discussed?

- Are all communities represented in such engagement activity, so that any redistributive effectives on noise exposure can be systematically addressed and consensus built as to the most socially optimal outcome(s)?

- Are such stakeholders and community groups engaged openly and transparently to establish trust? Is noise data made available on-line for those not able to attend such forums?

- Are there other avenues through which communities or other stakeholders can raise concerns with noise managers and/or make complaints?

- Are the concerns of those contacting an airport acknowledged? Are individuals provided with tailored responses relevant to their specific concern, rather than via template responses?

- Have you taken the time to question all assumptions about the need for the measure, and attempted to define it accurately? It can be helpful here to redefine the noise problem into a 'how might we question' that can more easily lead to actionable outcomes.

\section{Design of Options}

It can be helpful, when designing how the noise management measure is implemented, to conduct design approaches in two phases. A 'green-light' brainstorming phase in which there are no wrong answers can help to generate potentially novel solutions and things can be discarded too easily for being infeasible. Rather, greenlight brainstorming captures as many potential opportunities as possible and instead reviews them in a secondary-'red-light' brainstorming phase, where the proposed options are evaluated more critically. It can help to do this by using an evaluation matrix that may map options against criteria such as 'ease of implementation' and 'impact'. Doing so can help not just to determine what options have potential, but 
it can help to identify a pathway through which different options might be implementable. Stakeholder engagement and consultation is important at this stage as it helps to ensure that, for example, resident needs and perspectives are included in the design process, and so that more desirable outcomes can be achieved that are more likely to lead to what are perceived to be more acceptable results. Including residents in the decision-making process in this way also helps to embed the types of principles linked to perceptions of control and engagement as described in Chap. 11. When designing solutions, it can be helpful to think about questions such as:

- Are all stakeholders given the possibility of designing their own solutions to the required change, or informing in the discussion of the development of other options?

- Do stakeholders have the opportunity to work in collaboration with each other in identifying potential noise mitigation solutions?

- Are designs pre-informed by a set of criteria and objectives, for example by framing them within what is logistically feasible, safe, and regulatory compliant?

- Has time been taken to consider radically innovative solutions that may have been missed?

- Have combinations of solutions been considered rather than pursuing just one pillar of the Balanced Approach?

- Has the role of communication and engagement been considered as part of the design, for instance how non-acoustic factors may be impacted?

\section{Selection of Intervention Option}

With a range of design options developed, the most appropriate should be selected. This may include the use of a decision matrix that may include elements such as impact, desirability, feasibility and viability. Different options can be scored against each and the highest scoring option is thus the one that can be taken forward. It can be helpful here to consider long term solutions to the problem that could be implemented over longer-term periods through implementation pathways. Thus can be achieved by creating an idealised vision of what the solution to the given problem might look like in the future, and then working backwards from the idealised future state towards the present, thus creating a pathway of what needs to happen in turn for that vision to be realised. Doing so can reveal actions that need to be undertaken, stakeholders who need to be consulted with or informed, and more importantly the barriers that may need to be overcome. It can be helpful to think about questions such as the below when at this stage:

- Has modelling been carried out (ideally by an independent entity) to assess the impacts of the potential design options? Does this modelling include interdependencies?

- Are these results communicated to stakeholder forums for discussion? 
- Have all stakeholders been included in the discussion, even if they appear to be removed from the designed option (to help identify unintended consequences and trade-offs between communities)?

- Have the reasons why some options may not be feasible been communicated effectively?

- Have the results of any modelling, analysis and discussions been effectively disseminated to the public? So that there is a clear and transparent pathway that shows how the requirement for change was first raised, which options were considered, and why one, in particular, has been advocated.

- Have other complementary interventions been considered? For example, could an operational change be coupled with a change in land-use planning to enhance the predicted benefits?

- Have trials been carried out to verify modelling outcomes, and to perform analysis on the impacts on communities and other stakeholders?

- Do communities understand and value the metrics and dissemination tools used? Do you need to consider a different approach to communication?

\section{Implementation}

With a decision made as to how to address the problem, the solution to the problem can then be implemented. Example questions or thoughts that might be helpful at this stage might be:

- Have all stakeholders been made aware of the intervention in advance?

- Has the rationale for the chosen solution been explained to stakeholders rather than just the outcome of the decision making process itself?

- In order to demonstrate outcomes, have you considered if you need to move noise monitoring terminals, purchase new terminals, or make use of mobile terminals?

- Is regular feedback of the progress of the implementation made available to stakeholders?

- Have contingency plans been designed should the new procedure change and you need to fall back to the previous procedure?

- Do you have plans for on-going evaluation of the procedure, and plans for regular dissemination? This includes the collection of qualitative information.

\section{Post-Evaluation}

The saying 'you can't manage what you can't measure' is as true for noise management as it is for anything else. This enables performance to be assessed and for any potential changes to be made. It enables best practice to be extended to other areas and to add to the airports' knowledge of how to address noise problems in the 
future. Importantly, evaluation also enables the impacts of any noise impact abatement measures to be disseminated to stakeholders so that their success (or failure) can be demonstrated. Failure should not be hidden but rather embraced and made part of an on-going journey. Example helpful questions at this stage include:

- Have you committed to long term monitoring and evaluation and reporting to stakeholders?

- Do you communicate the procedure at engagement events?

- Do you have a long-term plan for the evaluation of the outcome of the intervention on non-acoustic factors, general acceptability of the decision and quality of life implications for local residents?

The above methodology is not intended to be a rigid approach to solving any noise management issue. Rather, the intention is to illustrate how this sort of design-led thinking can help to develop and deliver more effective noise management outcomes, but taking a considered approach to noise challenges. Taking the time to think about the challenge, understand and learn about it, embed communication and engagement into the decision-making process, making decisions based on empathy, and striving towards targeted outcomes that can be effectively evaluated to demonstrate success (or failure) to stakeholders, or to modify approaches in the future. In short, they help to show that the airport is not just seeking to do 'the right thing' but also to do that thing 'in the right way'.

\section{Lessons Learnt}

The implementation of ANIMA project during its four years of activity has allowed the participating partners to learn and ultimately share with the wider audience some key aspects.

First of all, the project definitively confirmed that the problem of aviation noise is not reduced only to a question of quantities of sonic pressure. Therefore, the adequate management to try to minimise noise in such a way so that it does not become, on the one hand, an issue that decreases the quality of life of many people, putting even their health at risk, and, on the other hand, an issue that limits the daily operation of airport infrastructure, must be approached jointly from different angles with the corresponding specialists and with the necessary tools, both hard and soft. It has also been found that since various disciplines as far apart as sociology and engineering participate, a "common language" is necessary - in some sorts, it could resemble an "Esperanto" for aviation noise management, which does not imply the abandonment of any of the languages already in use, but the creation of a third party that serves as a lingua franca, ensuring that all stakeholders, from top experts to local residents, can understand, at a certain level of acceptance, what aeronautical noise really implies.

Another great lesson is that, for different reasons (legislative, political, social, etc.), aviation noise is being taken very seriously by many organisations and institutions, and that many efforts are being devoted to avoid and minimise it from all possible 
points of view. It is precisely these efforts-led by airports, airlines and manufacturers - that, despite situations to be resolved and/or improved, there has been a large divergence between the vertiginous increase in the number of flights and the number of people affected by aviation noise. This divergence must be increased and can be increased in order to facilitate the growth of aviation and a positive relationship with airport environments and ANIMA can, hopefully, strongly help to do so by providing information, knowledge, methodology and best practice to be carried out by certain agents in the sector.

The development of the project itself-four years with multiple internal and external activities - and the in-depth knowledge of various experiences both in the investigation of new approaches and their subsequent application, has revealed that dealing with aviation noise is not only a question of shared visions, if not also of time, dedication and will. The struggle underpinning aviation noise and its impact on local communities as well as on airport growth needs to be prioritised by policymakers and addressing it should be one of the goals of the strategic policy agenda.

\section{References}

Brown T (2008) Design thinking. Harvard Business Review. https://doi.org/10.5749/minnesota/978 0816698875.003.0002

Downe L (2020) Good Services: How to design services that work, 1st edn. BIS Publishers. ISBN: 978-148 9,063,695,439

Goodheart B (2016) Design thinking for aviation safety. Aviat Bus J, 4

Hall A et al (2013) Future aircraft cabins and design thinking: optimisation vs. win-win scenarios. Propul Power Res 2(2):85-95. https://doi.org/10.1016/j.jppr.2013.04.001

Liedtka J (2015) Perspective: linking design thinking with innovation outcomes through cognitive bias reduction. J Prod Innov Manage 32:925-938. https://doi.org/10.1111/jpim.12163

McGowan AM, Bakula C, Castner R (2017) Lessons learned from applying design thinking in a nasa rapid design study in aeronautics. In: 58th AIAA/ASCE/AHS/ASC structures, structural dynamics, and materials conference, 2017

Turner Donald EIII (2017) A case study in design thinking applied through aviation mission support tactical advancements for the next generation (TANG). Calhoun. Available at: https://calhoun. nps.edu/handle/10945/56830

Open Access This chapter is licensed under the terms of the Creative Commons Attribution 4.0 International License (http://creativecommons.org/licenses/by/4.0/), which permits use, sharing, adaptation, distribution and reproduction in any medium or format, as long as you give appropriate credit to the original author(s) and the source, provide a link to the Creative Commons license and indicate if changes were made.

The images or other third party material in this chapter are included in the chapter's Creative Commons license, unless indicated otherwise in a credit line to the material. If material is not included in the chapter's Creative Commons license and your intended use is not permitted by statutory regulation or exceeds the permitted use, you will need to obtain permission directly from the copyright holder. 